\title{
Solar-terrestrial energy transfer during sunspot cycles and mechanism of Earth rotation excitation
}

\author{
Yavor Chapanov $^{1}$ and Daniel Gambis ${ }^{2}$ \\ ${ }^{1}$ Central Laboratory for Geodesy, Bulgarian Academy of Sciences \\ Akad. G. Bonchev Str. Bl.1, Sofia 1113, Bulgaria \\ email: chapanov@clg.bas.bg \\ ${ }^{2}$ SYRTE, Observatoire de Paris, CNRS, UPMC \\ 61, avenue de l'Observatoire, 75014 Paris, France \\ e-mail: daniel.gambis@obspm.fr
}

\begin{abstract}
The solar-terrestrial energy transfer, due to the total solar irradiance (TSI), solar wind and interplanetary magnetic field, has 11-year modulation during the sunspot cycles. Other oscillations of solar-terrestrial energy transfer are with periods of 22 and 45 year due to the magnetic reversal and equatorial solar asymmetry, which cause corresponding oscillations of all Earth systems, including climate and weather, atmosphere and ocean circulations, geomagnetic field and core processes. A part of this energy variation is transformed to oscillations of the Earth rotation. A model of indirect mechanism of Earth rotation excitation during sunspot cycles is proposed, which is based on global water circulation and periodical mass transfer between oceans and polar ice caps. The oscillations of the mean sea level (MSL) with periods 11, 22 and 45 years are determined by sea level data for the last two centuries from 13 maregraph stations. The necessary energy of water evaporation, corresponding to the observed MSL variations is provided by TSI oscillations with amplitudes between $0.2-0.5 \mathrm{~W} / \mathrm{m}^{2}$, determined by means of reconstructed time series of the TSI since 1610. The determined mean Universal Time (UT1) amplitudes, corresponding to the 22-year and 45-year cycles of the solar activity are $185 \mathrm{~ms}$ and $310 \mathrm{~ms}$.
\end{abstract}

Keywords. Sun: activity, solar-terrestrial relations, time

\section{Introduction}

The decadal variations of the Earth rotation are strongly affected by the cycles of the solar activity with a delay of about 1.5a. The explanation of the delayed UT1 response to the sunspot cycles is the appropriate change of the axial Earth moment of inertia, due to climatic variations, connected with the total solar irradiance change. The aim of this paper is to determine the mean amplitudes of the UT1 response to the solar activity cycles, according to the observed MSL variations and corresponding change of the Earth axial moment of inertia.

\section{Total solar irradiance variations}

Recently time series of Total Solar Irradiance (TSI) since 1610 was reconstructed by Lean et al.(1995)) and Lean (2000). These time series are shown in Fig. 1. The oscillations of the TSI with periods 11, 22 and 45 years (Fig.2, (a), (b), (c)) are determined by means of Fourier approximation and separation and combination of the oscillations with periods from the bands 10-12.5a for 11-year cycles; 19-25a for 22-year cycles and 34-56a for 45year cycles. The time series of 11-year and 45-year cycles of the TSI are determined 
by means of the second solution of TSI (Lean, 2000). Unfortunately, in this solution the 22-year component is with very small amplitude due to applied new corrections, while the first version contain rather significant 22-year oscillations. So, the 22-year cycles of the TSI are derived from the first reconstruction (Lean et al., 1995). The maximal amplitude of TSI oscillations with period $11 \mathrm{a}$ is $0.5 \mathrm{~W} / \mathrm{m}^{2}$ and $0.2 \mathrm{~W} / \mathrm{m}^{2}$ for 22 -year and 45 -year cycles.

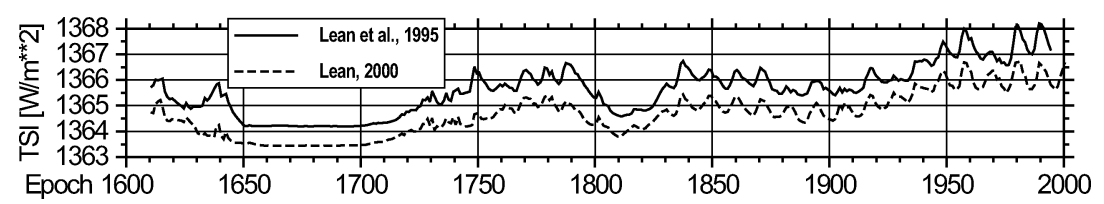

Figure 1. Time series of reconstructed Total Solar Irradiance (TSI) since 1610.

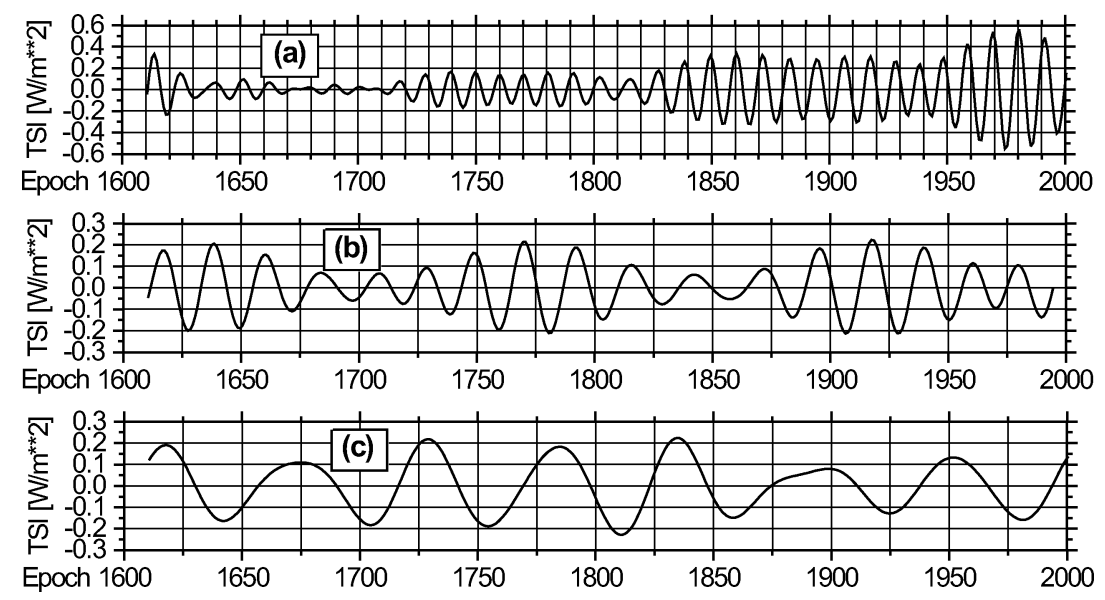

Figure 2. Time series of 11-year components of TSI (a), 22-year components of TSI (b) and 45-year components of TSI (c).

\section{Mean sea level oscillations}

The oscillations of the mean sea level with periods $11.25,22.5$ and 45 years are determined from maregraph data for the last two centuries by a model with fixed frequencies

$$
\begin{gathered}
\Delta L(t)=L_{0}+L_{1} t+\sum_{k=1}^{5} a_{k} \sin \frac{2 \pi}{T_{k}} t+b_{k} \cos \frac{2 \pi}{T_{k}} t, \\
A_{k}=\sqrt{a_{k}^{2}+b_{k}^{2}}
\end{gathered}
$$

where $\Delta L(t)$ are MSL variations for the epoch $t ; a_{k}, b_{k}$ are harmonically coefficients; $A_{k}$ are amplitudes of the oscillations with periods $T_{1}=1 \mathrm{a}, T_{2}=18.6 \mathrm{a}, T_{3}=11.25 \mathrm{a}$, $T_{4}=22.5 \mathrm{a}$ and $T_{5}=45 \mathrm{a}$. The estimated amplitudes of MSL oscillations at 13 maregraph stations are given in Table 1 . The corresponding mean amplitudes $A_{11}, A_{22.5}$ and $A_{45}$ are $6.9 \pm 2 \mathrm{~mm} ; 10.6 \pm 3 \mathrm{~mm}$ and $8.9 \pm 2 \mathrm{~mm}$.

\section{Conclusions}

- The 11-year, 22-year and 45-year solar activity cycles strongly affect earth climatic variations by the variations of the total solar irradiance with maximal amplitudes between $0.2-0.5 \mathrm{~W} / \mathrm{m}^{2}$. These variations provide additional amount of energy $(\sim 1017 \mathrm{MJ}$ for the 
Table 1. Amplitudes $A_{11}, A_{22.5}, A_{45}$ of the 11-year, 22.5-year and 45-year oscillations of the mean sea level for some maregraph stations. Values less than $3 \mathrm{~mm}$ are removed.

\begin{tabular}{lcccc}
\hline Station & Period & $\mathbf{A}_{\mathbf{1 1}}[\mathbf{m m}]$ & $\mathbf{A}_{\mathbf{2 2 . 5}}[\mathbf{m m}]$ & $\mathbf{A}_{\mathbf{4 5}}[\mathbf{m m}]$ \\
\hline Balboa & $1908-1996$ & 11.7 & 9.6 & 6.0 \\
Barencburg & $1948-2006$ & 8.3 & 18.6 & 11.0 \\
Brest & $1807-2005$ & 4.4 & - & - \\
Fremantle & $1897-1990$ & 4.9 & 11.0 & 6.3 \\
Genova & $1884-1998$ & 4.7 & 10.9 & 11.3 \\
Ijmuiden & $1871-2006$ & 6.5 & 6.3 & 6.3 \\
Harlingen & $1865-2005$ & 7.4 & 1.5 & - \\
Honolulu & $1905-2006$ & 6.4 & 8.3 & 12.4 \\
Maassluis & $1848-2006$ & 6.2 & 8.5 & - \\
Mumbai & $1878-1994$ & 6.1 & 17.7 & 3.7 \\
Prince Rupert & $1909-2006$ & 14.2 & 8.7 & 9.8 \\
Stockholm & $1774-2001$ & 9.1 & $10.6 \pm 3$ & $8.9 \pm 2$ \\
Sydney & $1886-1994$ & 4.5 & & \\
\hline Mean & & $6.9 \pm 2$ & & \\
\hline
\end{tabular}

Notes:

${ }^{1}$ The mean values are calculated after removing one minimal and one maximal amplitudes.

whole Earth and 130-260MJ per square meter on the equator) during these cycles. This energy is capable to evaporate 50-1001 of water from 1 square meter equatorial ocean surface and 20-401 of water from 1 square meter ocean surface from the north latitudes.

- The periodical UT1 variations due to solar cycles are provided by additional water evaporation and global mean sea level oscillations, synchronized with the global water redistribution and corresponding change of the ice thickness at the polar caps. These events cause periodical change of the Earth axial moment of inertia and periodical oscillations of the Earth rotation, according the law of angular momentum conservation. The amplitude of $1 \mathrm{~mm}$ of the mean sea level oscillation with periods 11, 22 and 45 years corresponds to UT1 oscillations with the same periods and amplitudes $12.5 \mathrm{~ms}, 25 \mathrm{~ms}$ and $50 \mathrm{~ms}$.

- The oscillations of the mean sea level with periods $11.25,22.5$ and 45 years at 13 maregraph stations are determined by a model with fixed frequencies. The corresponding mean amplitudes of these oscillations are $6.9 \pm 2 \mathrm{~mm} ; 10.6 \pm 3 \mathrm{~mm}$ and $8.9 \pm 2 \mathrm{~mm}$. The previously estimated UT1 response to 11-year sunspot cycles with amplitude $60 \mathrm{~ms}$ need global water redistribution from oceans to the polar caps, corresponding to "pure" mean sea level oscillation with amplitude of $4.8 \mathrm{~mm}$ (Chapanov \& Gambis 2008). The rest of $2.1 \mathrm{~mm}$ to the observed 11-year MSL amplitude is redistributed over the continents. So, according the above method of MSL oscillations determination with fixed frequencies, the proportion between the redistributed water over the polar caps and continents is $70 \%$ to $30 \%$. The effectiveness of $70 \%$ means that the effective MSL amplitudes $A_{22.5}$ and $A_{45}$ are $7.4 \mathrm{~mm}$ and $6.2 \mathrm{~mm}$, so the expected mean UT1 amplitudes, corresponding to the 22 -year and 45 -year cycles of the solar activity, are $185 \mathrm{~ms}$ and $310 \mathrm{~ms}$.

\section{Acknowledgements}

The work was supported by grant No. DO 02-275 with Bulgarian NSF.

\section{References}

Chapanov Ya. \& Gambis, D. 2008, Journées 2008 “Syst. de ref. spat.-temp.", Dresden, 131-132 Lean, J., Beer, J., \& Bradley, R. 1995, Geophys. Res. Let., 22, 3195-3198

Lean, J. 2000, Geophys. Res. Let., 27, 2425-2428 